\title{
Gamificação, elementos de jogos e estratégia: uma matriz de referência
}

\author{
Gamification, elements of games and strategy: a reference matrix
}

\author{
Amanda Cristina Santos Costa \\ Bacharel em Gestão da Informação pela Universidade Federal do Paraná - UFPR. \\ Assistente de Entregas ExxonMobil. \\ E-mail:amandacostagi@gmail.com
}

Patricia Zeni Marchiori

Doutora em Ciências da Comunicação pela Universidade de São Paulo - USP.

Professora do Departamento de Ciência e Gestão da Informação da Universidade Federal do Paraná - UFPR

pzeni@ufpr.br

\begin{abstract}
Resumo
Situando-se as pesquisas de gamificação - entendida como a utilização de elementos de design de jogos em contextos que não são de jogos - nas discussões referentes à arquitetura de informação, o estudo apresenta uma matriz de referência para aplicações de gamificação a partir da sistematização de elementos de jogos (dinâmicas, mecânicas e componentes) e da busca de relações com segmentos/áreas de aplicação da gamificação. Além da pesquisa bibliográfica exploraram-se técnicas da Teoria Fundamentada nos Dados (Grounded Theory) para coleta, preparação e análise de dados. Matrizes intermediárias permitiram discutir aspectos relativos aos componentes, mecânicas e dinâmicas de jogo e os distintos segmentos/áreas identificados. A matriz principal pode servir de modelo de referência para aplicações de gamificação destacando-se que diferentes elementos de jogos podem ser combinados de diversas maneiras, e que cada segmento/área apresenta um potencial de combinações que dependem da clareza dos objetivos de negócio. Um exemplo nesta direção seria o uso da dinâmica de "narrativas" no segmento Educação e Treinamento devido ao estímulo, motivação e engajamento que estas podem promover no processo de ensino/aprendizagem. No contexto da arquitetura de informação, as mecânicas de jogo são elementos críticos para o engajamento, pois estruturam o estilo do jogo e atuam diretamente na percepção do usuário. Estudos futuros podem agregar a esta matriz informações prévias dos usuários - as quais afetariam o desenvolvimento das aplicações de gamificação - assim como informações derivadas da atividade dos jogadores, enquanto recortes voltados a uma proposta estratégica mais ampla.
\end{abstract}

Palavras-chave: Elementos de gamificação. Segmentos para gamificação. Mecânica de jogo. Dinâmica de jogo. Componentes de jogo.

\begin{abstract}
Investigations about gamification - understood as the use of game design elements in no game contexts - could be suitable to the issue of information architecture as far as the engagement aspect is concerned. The study presents a reference matrix for gamification applications based on a framework of game elements (dynamics, mechanics and components) seeking relations with segments/application areas that could make use of the gamification process. Besides the bibliographical research, some Grounded Theory techniques were used to gather, prepare and analyze data. Intermediate matrices allowed the discussion about aspects related to components, mechanics and game dynamics and the different segments/areas identified. The main matrix may serve as a reference model for gamification applications bearing in mind that different sets of elements may be combined in various ways; and that each segment/area has a potential set of combinations that depend on the clarity of business objectives. For example, the use of the "narratives" dynamics in the Education and Training segment could help not only the players, but also the developers regarding the stimulus, motivation and engagement needed to promote a teaching/learning process. Within the context of information architecture, the game mechanics is the critical element for engagement underpinning the play style and working directly on the user's perception. Future studies may add to this matrix previous information from users - which would affect the development of gamification applications - as well as information derived from the activity of the players, as cutouts towards a wide strategic proposal.
\end{abstract}

Keywords: Gamification elements. Segments for gamification. Game mechanics. Game dynamics. Game components.

InCID: R. Ci. Inf. e Doc., Ribeirão Preto, v. 6, n. 2, p. 44-65, set. 2015/fev. 2016.

DOI: 10.11606/issn.2178-2075.v6i2p44-65 


\section{Introdução}

Entendida comumente como a utilização de elementos de design de jogos em contextos que não são de jogos (DETERDING, 2011), a gamificação pode ser definida de forma mais consistente como sendo uma estratégia apoiada na aplicação de elementos de jogos para atividades non-game $e^{l}$ que é utilizada para influenciar e causar mudanças no comportamento de indivíduos e grupos (BUNCHBALL INC., 2010). Para Deterding et al. (2013), a gamification é um fenômeno da Tecnologia da Informação (TI) cujo conceito é considerado por alguns apenas um modismo, uma simples buzzword e, por outros, uma solução real para diversos problemas organizacionais.

Uma das possíveis interfaces entre a gamificação e a Ciência da Informação situa-se no contexto das pesquisas em arquitetura de informação. Para Toms (2002), usuários de informação no século XXI não apenas buscam por informação, mas "submergem" em volumes crescentes de dados. Para a autora, a arquitetura de informação - entre outros componentes buscaria contribuir não apenas para a interatividade do usuário, mas para aprimorar sua experiência com a informação. Nesta perspectiva, o engajamento das pessoas com a tecnologia, na visão de Quesenbery (2003) extrapola a usabilidade (como entendida tradicionalmente), uma vez que está relacionada com os aspectos emocionais do indivíduo. Baseando-se em teorias de interatividade e de processamento de informação e a partir da seleção de quatro tipo de aplicações computacionais (compras online, busca na web, webcasting ${ }^{2}$ e videogames), O'Brien e Toms (2008) propõem um modelo de engajamento de usuários. Nesta pesquisa, estudos listados pelas autoras revelam que o engajamento se relaciona diretamente com as características das mídias, assim como percebidas pelos usuários, tais como: a apresentação; os níveis de controle; os desafios propostos; a variedade e possibilidade de escolha; e, o feedback (O’BRIEN; TOMS, 2008). Controle, desafio e feedback são considerados dimensões intrínsecas dos jogos e a maneira de engajar os usuários em tais dimensões define a efetividade da plataforma (GARRIS; AHLERS; DRISKELL, 2002).

Um dos mais conhecidos exemplos de gamificação é o Foursquare, (DETERDING et al., 2011). Lançado em 2009 como um aplicativo de rede social de compartilhamento local,

\footnotetext{
${ }^{1}$ Forma de entretenimento que não tem um vencedor ou conclusão (IWATA KEYNOTE apud FAHEY, 2005).

2 Webcast é o processo de transmissão de conteúdo multimídia (áudio e vídeo) através da internet. Para isto, é utilizada a tecnologia de streaming, onde a partir de uma única fonte de dados são gerados áudio e vídeo e transmitido simultaneamente para inúmeros clientes (CELEPAR INFORMÁTICA DO PARANÁ. Webcast CELEPAR: descrição do serviço. c2007. Disponível em: 〈http://webcast.pr.gov.br/〉. Acesso em: 20 ago. 2015).
} 
logo se tornou um modelo para futuros projetos de gamification (MCCORMICK, 2013). Outro caso de sucesso quando da implantação de processos de gamificação é o da fabricante mundial de calçados esportivos e vestuário Nike. Com o lançamento do Nike+, em 2008, a companhia convenceu mais de 1,8 milhão de corredores a utilizarem seus produtos. $\mathrm{O}$ aplicativo desenvolvido permite a captura de dados como distância, ritmo e calorias queimadas através de um sensor GPS conectado ao iPod. Ao completarem desafios e baterem seus próprios recordes de corrida os usuários ganham recompensas. Além disso, após os treinos, os corredores podem acessar a loja online para fazer upload de seus dados, acompanhar as suas estatísticas, definir metas, participar de desafios, e se conectar com colegas corredores (BUNCHBALL INC., 2010). Programas de milhagem também utilizam a gamificação e, cerca de 120 milhões de pessoas em todo o mundo fazem parte desse tipo programas oferecidos por companhias aéreas, onde elementos de jogos são inseridos na forma de pontos e níveis. Neste tipo de aplicação os clientes acumulam milhas (pontos) para cada voo realizado, aumentando seu status (nível) de prata para ouro, por exemplo (BUNCHBALL INC., 2010).

No meio empresarial, a gamificação tornou-se popular por trazer uma abordagem de envolvimento e produtividade dos funcionários ( $\mathrm{WEBB}^{3}, 2013$ apud SCHÖNEN, 2014). Além do mundo dos negócios, a gamificação é aplicável a diversos outros segmentos/áreas tais como (BUNCHBALL INC., 2012):

a) Saúde: auxiliando na contenção de custos, programas de obesidade, cessação do tabagismo;

b) Educação: engajando estudantes com elementos de jogos em e-learning;

c) Políticas públicas e governo: incentivando melhorias na educação e no exercício da cidadania.

Em estudo de 2011, o Gartner Group revelou que a gamification apresentava um movimento de ascensão prevendo-se um pico de expectativa para os próximos 5 a 10 anos (CUCCUREDDU, 2011). Porém, em 2014, nova pesquisa apontou que oitenta por cento das aplicações de gamificação não cumpririam os objetivos de negócio devido, principalmente, a problemas no design (GARTNER GROUP, 2012). Neste mesmo ano, Nicholson (2012) destacou que parte significativa das gamificações ainda se caracteriza como a aplicação simples de pontos, medalhas, e de ranking de líderes sem qualquer compromisso com a visão de longo

\footnotetext{
${ }^{3}$ WEBB, E. N. Gamification: when It works, when it doesn't. In: MARCUS, A. (Ed.). Design, user experience, and usability: health, learning, playing, cultural, and cross-cultural user experience. Springer Science, 2013. p. 608-614. Disponível em: 〈http://dx.doi.org/ 10.1007/978-3-642-39241-2_67>. Acesso em: 02 set. 2015.
} 
prazo. $\mathrm{O}$ autor ainda alerta que a gamification tem recebido críticas negativas significativas por aqueles que estudam jogos. Primeiramente, ao se colocar o termo "jogo" (game) como prefixo de gamification há um reforço de que toda a atividade vai se tornar uma experiência envolvente quando, na realidade, a gamification normalmente utiliza apenas a parte menos interessante de um jogo como, por exemplo, o sistema de pontuação. O termo "pointsification" tem sido sugerido como um rótulo para sistemas com estas características. Um argumento em defesa desta diferenciação pondera que a gamificação é uma forma de jogo com objetivos e estrutura (MARONEY, 2001), e que a gamificação baseada em pontos se concentra apenas nos objetivos, ignorando o próprio jogo.

Porém, se reconhece o potencial da gamificação para a criação de conteúdo em conjunto com os usuários; no incentivo ao uso dos produtos e serviços; no aumento da visibilidade da marca; e, no reforço dos comportamentos desejados dos usuários. Contudo, é vital que os responsáveis pela implantação tenham informações sobre o público alvo, necessidades e os elementos de jogos existentes (PRIEBATSCH, 2010; KUUTTI, 2013).

A informação assume, no contexto da gamificação, um caráter estratégico em dois momentos. Em um primeiro momento, os responsáveis pelo processo devem demandam informações que apoiem um projeto de gamificação voltado para um produto/serviço informacional que assumirá um valor estratégico sob o enfoque da competitividade. Em um segundo momento, considerando-se que o jogo já esteja alinhado uso ótimo dos elementos de jogos, a organização poderá coletar a informação proveniente dos “jogadores" e analisá-las conforme os objetivos da organização.

Quanto aos elementos de jogos, Werbach e Hunter (2012) identificaram dinâmicas, mecânicas e componentes como categorias válidas para desenvolvimento e estudos na temática. Essa tríade se organiza em ordem decrescente de abstração, de modo que cada mecânica se liga a uma ou mais dinâmicas, e cada componente a uma ou mais mecânicas ou dinâmicas (KUUTTI, 2013). Neste contexto, é preciso levar em conta o segmento ou área em que a gamificação será posta em ação. Para o Instituto Brasileiro de Geografia e Estatística, um segmento pode ser entendido como uma categoria de unidade de produção separada das demais de acordo com a atividade que desenvolve. Os segmentos são estabelecidos com base na similaridade de funções produtivas (insumos, tecnologia, processos), características dos bens e serviços e finalidades de uso como, por exemplo, a administração pública e serviços, a saúde e educação (INSTITUTO..., 2007).

InCID: R. Ci. Inf. e Doc., Ribeirão Preto, v. 6, n. 2, p. 44-65, set. 2015/fev. 2016. 
Gamificação, elementos de jogos e estratégia: uma matriz de referência

Assim, pretende-se que esse estudo contribua com a área de Gestão da Informação a partir da abordagem de relações entre elementos de jogos e suas possíveis aplicações em determinados segmentos ou áreas, auxiliando os responsáveis pelos processos de gamificação na identificação dos elementos de jogos mais adequados para o seu negócio, orientando-os no planejamento de jogos e, potencialmente, em futuras comprovações empíricas que venham a adicionar novos segmentos e elementos, devido ao dinamismo da temática. O presente estudo limita-se à análise e sistematização dos elementos de jogos aplicáveis a gamificações voltada às finalidades e a relação com o segmento/área de aplicação na qual foram utilizadas técnicas da Teoria Fundamentada em Dados (TFD).

\section{Elementos de jogos}

Werbach e Hunter (2012) identificaram três tipos de elementos (dinâmicas, mecânicas e componentes) como categorias aplicáveis aos estudos e desenvolvimento da gamificação. Tais categorias são organizadas em ordem decrescente de abstração de modo que cada mecânica se liga a uma ou mais dinâmicas, e cada componente a uma ou mais mecânicas ou dinâmicas.

As dinâmicas de jogos representam o mais alto nível de abstração de elementos do jogo (Quadro 1). São os temas em torno do qual o jogo se desenvolve, assim como aspectos do quadro geral do sistema de jogo levados em consideração, mas que não fazem parte diretamente do jogo. Esses elementos mostram quais são as forças subjacentes que existem em jogos (WERBACH; HUNTER, 2012).

As dinâmicas, portanto, representam as interações entre o jogador e as mecânicas de jogo e compõem os aspectos do quadro geral de uma gamificação. Devem ser gerenciadas, mas não são explicitadas obrigatoriamente no jogo (WERBACH; HUNTER, 2012). 
Quadro 1 - Dinâmicas de jogo - Conceituações

\begin{tabular}{|cl|}
\hline Dinâmicas & \multicolumn{1}{c|}{ Descrição } \\
\hline Emoções & $\begin{array}{l}\text { Jogos podem criar diferentes tipos de emoções, especialmente a da diversão (reforço } \\
\text { emocional que mantém as pessoas jogando) }\end{array}$ \\
Narrativa & $\begin{array}{l}\text { Estrutura que torna o jogo coerente. A narrativa não tem que ser explícita, como uma } \\
\text { história em um jogo. Também pode ser implícita, na qual toda a experiência tem um } \\
\text { propósito em si }\end{array}$ \\
Progressão & $\begin{array}{l}\text { Ideia de dar aos jogadores a sensação de avançar dentro do jogo } \\
\text { Relacionamentos }\end{array}$ \\
$\begin{array}{l}\text { Refere-se à interação entre os jogadores, seja entre amigos, companheiros ou } \\
\text { adversários }\end{array}$ \\
Restrições & Refere-se à limitação da liberdade dos jogadores dentro do jogo \\
\hline
\end{tabular}

Fonte: Traduzido, com adaptações de Werbach; Hunter, 2012

As mecânicas se referem aos elementos mais específicos que levam às ações também mais específicas (Quadro 2). Elas orientam as ações dos jogadores em uma direção desejada delimitando que o jogador pode ou não fazer dentro do jogo (ERIKSSON, MUSIALIK, WAGNER, 2012).

Quadro 2 - Mecânicas de Jogo - Conceituações

\begin{tabular}{|cl|}
\hline Mecânicas & Descrição \\
\hline $\begin{array}{c}\text { Aquisição de } \\
\text { recursos }\end{array}$ & O jogador pode coletar itens que o ajudam a atingir os objetivos \\
$\begin{array}{c}\text { Avaliação } \\
\text { (Feedback })\end{array}$ & A avaliação permite que os jogadores vejam como estão progredindo no jogo \\
Chance & $\begin{array}{l}\text { Os resultados de ação do jogador são aleatórios para criar uma sensação de surpresa e } \\
\text { incerteza }\end{array}$ \\
$\begin{array}{cl}\text { Cooperação e } \\
\text { competição }\end{array}$ & Cria-se um sentimento de vitória e derrota \\
Desafios & Os objetivos que o jogo define para o jogador \\
Recompensas & O benefício que o jogador pode ganhar a partir de uma conquista no jogo \\
Transações & Significa compra, venda ou troca de algo com outros jogadores no jogo \\
& $\begin{array}{l}\text { Cada jogador no jogo tem seu próprio tempo e oportunidade para jogar. Jogos tradicionais, } \\
\text { como jogos de cartas e jogos de tabuleiro muitas vezes dependem de turnos para manter o }\end{array}$ \\
Turnos & $\begin{array}{l}\text { equilíbrio no jogo, enquanto muitos jogos de computador modernos trabalham em tempo } \\
\text { real }\end{array}$ \\
Vitória & O “estado" que define ganhar o jogo \\
\hline
\end{tabular}

Fonte: Traduzido, com adaptações de Werbach; Hunter, 2012

As mecânicas viabilizam o funcionamento do jogo e orientam as ações do jogador; e, dependendo da mecânica utilizada, os jogos podem ter uma variedade ampla de estilos (ZICHERMANN; CUNNINGHAM, 2011). Vários mecanismos podem estar incluídos em uma dinâmica como, por exemplo, feedback e recompensas - os quais podem dar uma sensação de 
Gamificação, elementos de jogos e estratégia: uma matriz de referência

progressão no jogo. Assim, cada mecânica é uma forma de atingir uma ou mais das dinâmicas descritas. Um evento aleatório, tal como um prêmio que aparece sem aviso, pode tanto estimular o senso de diversão e curiosidade dos jogadores como ser uma forma de obter novos participantes ou manter os jogadores mais experientes envolvidos (WERBACH; HUNTER, 2012).

Os componentes são aplicações específicas visualizadas e utilizadas na interface do jogo (Quadro 3). Este é o nível mais concreto dos elementos de jogos e, assim como uma mecânica se liga com uma ou mais dinâmicas, vários componentes podem fazer parte de uma mecânica.

Quadro 3 - Componentes de Jogo - Conceituação

\begin{tabular}{|c|c|}
\hline Componentes & Descrição \\
\hline Avatar & Representação visual do personagem do jogador \\
\hline Bens virtuais & $\begin{array}{l}\text { Itens dentro do jogo que os jogadores podem coletar e usar de forma virtual e não } \\
\text { real, mas que ainda tem valor para o jogador. Os jogadores podem pagar pelos itens } \\
\text { ou moeda do jogo ou com dinheiro real }\end{array}$ \\
\hline Boss & $\begin{array}{l}\text { Um desafio geralmente difícil no final de um nível que tem deve ser derrotado, a } \\
\text { fim de avançar no jogo }\end{array}$ \\
\hline Coleções & $\begin{array}{l}\text { Formadas por itens acumulados dentro do jogo. Emblemas e Medalhas são } \\
\text { frequentemente parte de coleções }\end{array}$ \\
\hline Combate & Disputa que ocorre para que o jogador derrote oponentes em uma luta \\
\hline Conquistas & Recompensa que o jogador recebe por fazer um conjunto de atividades específicas \\
\hline $\begin{array}{l}\text { Conteúdos } \\
\text { desbloqueáveis }\end{array}$ & $\begin{array}{l}\text { A possibilidade de desbloquear e acessar certos conteúdos no jogo se os pré- } \\
\text { requisitos forem preenchidos. O jogador precisa fazer algo específico para ser } \\
\text { capaz de desbloquear o conteúdo }\end{array}$ \\
\hline Emblemas/medalhas & Representação visual de realizações dentro do jogo \\
\hline Gráfico Social & $\begin{array}{l}\text { Capacidade de ver amigos que também estão no jogo e ser capaz de interagir com } \\
\text { eles. Um gráfico social torna o jogo uma extensão de sua experiência de rede } \\
\text { social. }\end{array}$ \\
\hline Missão & $\begin{array}{l}\text { Similar a "conquistas". É uma noção de jogo de que o jogador deve fazer executar } \\
\text { algumas atividades que são especificamente definidas dentro da estrutura do jogo }\end{array}$ \\
\hline Níveis & $\begin{array}{l}\text { Representação numérica da evolução do jogador. O nível do jogador aumenta à } \\
\text { medida que o jogador se torna melhor no jogo. }\end{array}$ \\
\hline Pontos & Ações no jogo que atribuem pontos. São muitas vezes ligadas a níveis \\
\hline Presentes & $\begin{array}{l}\text { A possibilidade distribuir ao jogador coisas como itens ou moeda virtual para } \\
\text { outros jogadores }\end{array}$ \\
\hline Ranking & $\begin{array}{l}\text { Lista jogadores que apresentam as maiores pontuações/conquistas/itens em um } \\
\text { jogo }\end{array}$ \\
\hline Times & Possibilidade de jogar com outras pessoas com mesmo objetivo \\
\hline
\end{tabular}

Fonte: Traduzido, com adaptações, de Werbach; Hunter, 2012

InCID: R. Ci. Inf. e Doc., Ribeirão Preto, v. 6, n. 2, p. 44-65, set. 2015/fev. 2016. 
Werbach e Hunter (2012) salientam que os componentes descritos acima podem assumir diversas combinações, e essa escolha deve ser feita com base no que atende mais adequadamente as demandas de um determinado contexto. Combinar as dinâmicas, mecânicas e componentes de forma que sejam efetivas para um determinado objetivo é a tarefa central de um projeto de gamificação.

Deterding (2012) alerta que desenvolvedores que acreditarem que "gamificar" é a simples adição de recompensas e incentivos com pontos e medalhas dentro de um sistema, estão destinados a fracassar em suas aplicações.

\section{Procedimentos metodológicos: técnicas da teoria fundamentada nos dados}

De acordo com Corbin e Strauss (1990), a Teoria Fundamentada nos Dados (TFD) tem como objetivo identificar, desenvolver e relacionar conceitos a partir da coleta e análise sistemática de dados. Os dados a serem coletados e analisados podem derivar de qualquer tipo de fonte que possa esclarecer as questões em estudo, sejam elas, entrevistas, observações, documentos, fitas de vídeo, jornais, cartas ou livros (CORBIN; STRAUSS, 1990).

Charmaz (2006) define a TFD como um método de condução de pesquisa qualitativa que se concentra na criação de modelos conceituais ou teorias através da construção de análise indutiva dos dados. As categorias analíticas são provenientes diretamente dos dados e o método favorece uma análise baseada na descrição de categorias ao invés de ideias preconcebidas e teorias já existentes.

A TDF inicia-se com a seleção e coleta de dados e, em seguida, são delimitados os pontos-chave - entendidos como uma série de códigos que são extraídos das fontes - e que representam o assunto de forma sucinta e que são agrupados em conceitos semelhantes ao se examinarem proporções mais amplas do texto que apresentem significado analítico mais relevante. A partir destes conceitos, categorias são formadas, dando base para a criação de uma teoria (CORBIN; STRAUSS, 1990). A TFD é, portanto, um método de análise capaz de promover o desenvolvimento de interpretações preliminares sobre os dados pela codificação e categorização, que permitem a construção de um controle sistemático dessas categorias verificando-se a variação e relações entre elas (CHARMAZ, 2006). 
Vale ressaltar que este trabalho não pretendeu elaborar uma teoria a partir da TFD, ainda que este seja seu objetivo macro (CORBIN; STRAUSS, 1990). A adoção de técnicas da TFD nesta investigação teve como finalidade auxiliar a construção de uma matriz que relacione os elementos de jogos e segmentos/áreas em que a gamificação se aplica, uma vez que as categorias podem surgir das fontes consultadas. As etapas utilizadas se dividem, portanto, em uma etapa de coleta de dados seguida de uma etapa de codificação, a qual foi composta a partir duas técnicas diferentes da TFD - codificação aberta e codificação axial.

\section{Etapa de coleta}

Esta etapa prevê a seleção de um ou vários canais de comunicação; a definição do(s) idioma(s); a definição do arco temporal para a coleta dos documentos; e, a seleção e exploração dos tipos documentais disponíveis. A pesquisa foi realizada com base em materiais recuperados a partir de expressões de busca inseridas em agregadores e bases de dados científicas e no Google Scholar (Quadro 4). O intervalo de data de publicação foi fixado em 2000 até 2014, uma vez que nenhuma destas bases apresentou resultados anteriores a 2000.

Quadro 4 - Lista de termos utilizados para busca do corpus - fase de coleta (maio 2014)

\begin{tabular}{|c|c|c|}
\hline \multicolumn{3}{|c|}{ Expressões de Busca } \\
\hline Português & Inglês & Espanhol \\
\hline Elementos de gamificação & Gamification elements & Elementos del gamificación \\
\hline Mecânica de gamificação & Gamification mechanics & Mecânica del gamificación \\
\hline Dinâmica de gamificação & Gamification dynamics & Dinámica del gamificación \\
\hline Técnicas de gamificação & Gamification techniques & Técnicas del gamificación \\
\hline Elementos de jogo & Game elements & Elementos del juego \\
\hline Mecânica de jogo & Game mechanics & Mecânica del juego \\
\hline Dinâmica de jogo & Game dynamics & Dinámica del juego \\
\hline Ontologia de gamificação & Gamification ontology & Ontología del gamificación \\
\hline Taxonomia de gamificação & Gamification taxonomy & Taxonomía del gamificación \\
\hline
\end{tabular}

Fonte: Os autores

Determinou-se como critério a pesquisa pelas expressões de busca que foram apliadas aos campos título, resumo e texto completo. Ao se buscar uma expressão (tal como "Game Elements") se fez necessário agregar a palavra gamificação (e suas respectivas traduções para o inglês e o espanhol). As expressões foram inseridas sem recursos avançados de busca, como 
por exemplo, a inserção de aspas. A busca retornou 138 materiais, todos no idioma inglês. Um segundo conjunto de critérios foi aplicado ao material recuperado:
a) ser um artigo completo;
b) ser um estudo empírico;
c) ser um estudo com o segmento/área identificado; e,
d) ser um estudo com elementos de jogos definidos.

Após a aplicação destes critérios, o corpus resultante foi de 54 itens (dos 138 iniciais).

\section{Etapa de codificação/análise dos dados}

Durante a etapa de codificação ocorre o processo de definição sobre "o que tratam os dados" e, ao contrário de pesquisas quantitativas que aplicam categorias pré-concebidas, a TFD cria códigos qualitativos derivados dos dados para definir o que se encontra nestes próprios dados (CHARMAZ, 2006). Assim, os códigos são emergentes e se desenvolvem na medida em que o pesquisador estuda os seus dados. O processo de codificação pode levar o pesquisador a temas não previstos (CHARMAZ, 2006) e, de acordo com Corbin e Strauss (1990), nos códigos identificados encontram-se conceitos que pertencem ao mesmo fenômeno e que podem ser agrupados para formar categorias. Porém, nem todos os conceitos se tornam categorias. Categorias são mais elevadas em nível e mais abstratas do que os conceitos. Elas são geradas pelo mesmo processo analítico de fazer comparações para destacar semelhanças e diferenças que são usadas para produzir conceitos de nível mais baixo.

A TFD sugere uma série de técnicas de codificação variadas para analisar dados, as quais apresentam distintos níveis de complexidade e foco. A forma escolhida de codificação define a maneira de seleção e classificação dos dados que foram separados para iniciar a parte de análise. Diante do exposto, assumiram-se dois tipos de codificação (CORBIN; STRAUSS, 1990):

a) Codificação Aberta: visa identificar, analisar e categorizar dados brutos. Os dados recebem um código do pesquisador e são organizados em categorias de acordo com suas propriedades e semelhanças;

b) Codificação Axial: visa sintetizar e explicar os segmentos de dados extraídos da codificação aberta. São utilizados os códigos mais significantes ou mais frequentes para 
representar os dados como um todo. O escopo desta etapa é identificar as interligações entre as categorias com base nas suas propriedades.

Para rodada da codificação aberta foi elaborada uma planilha de dados para sistematizar uma primeira identificação de categorias. Embora inicialmente se considerasse a existência e identificação de segmentos/áreas, tipos de usuários e elementos de jogos como categorias que possam emergir dos materiais selecionados, procurou-se não predefinir tais categorias, uma vez que o objetivo da codificação aberta é identificar, analisar e categorizar dados brutos sem ideias preconcebidas.

Durante esta etapa, nove dos 54 materiais recuperados não apresentaram características detalhadas sobre a aplicação de gamificação, dificultando a identificação dos elementos de jogos. Utilizou-se, então a técnica de encadeamento para trás (Backward Chaining), a qual se caracteriza por consultar as fontes e referências citadas no material primeiramente consultado (ELLIS, 1989). A utilização desta técnica resultou sete itens somando 52 estudos para as etapas de codificação. Vale ressaltar que em alguns materiais, identificou-se mais de uma aplicação de gamificação. Decidiu-se, portanto, trabalhar a análise por números de casos (61 no total).

A codificação axial corresponde a um tipo de codificação que trata a categoria como um eixo em torno do qual o pesquisador traça relações e especifica as dimensões dessa categoria. Um dos principais objetivos da codificação axial é trazer os dados juntos novamente em um todo coerente. Esta codificação relaciona categorias à subcategorias, especifica as propriedades e dimensões de uma categoria, e reagrupa os dados para dar coerência à análise emergente (CHARMAZ, 2006). Como parte da codificação axial, elaboraram-se duas matrizes com as (macro) categorias finais identificadas na codificação aberta. Definidas as categorias, estas foram retrabalhadas em novas planilhas, permitindo a análise de relações, coocorrências e eventuais discrepâncias. 


\section{Análise e discussão dos resultados}

Uma verificação preliminar dos dados da codificação aberta resultou nas as seguintes variáveis: ano de aplicação da gamificação, tipo da aplicação, segmento/área de aplicação, objetivo, público alvo e elementos de jogos separados em dinâmica, mecânica e componente.

Os pontos-chave identificados de cada variável na etapa de codificação aberta foram organizados em categorias, de acordo com suas propriedades e semelhanças (Quadro 5).

Quadro 5 - Codificação Aberta: Variáveis e Pontos-Chave

\section{Variável}

Pontos-Chave

Ano da Aplicação 2010, 2011, 2012, 2013 e 2014

Tipo da Aplicação Modelo de ensino, aplicativo móvel, aplicação web, sistema de informação interno, plataforma e-learning.

Motivar estudantes, motivar estudo, engajar e motivar usuário, manter usuários,

Objetivos aumentar vendas, manter clientes, educar usuários, treinar usuários, engajar e motivar funcionários, treinar funcionários

Público Alvo Clientes, estudantes, funcionários, fãs, organização, usuários, usuários experientes e crianças

Segmento/Área Colaboratividade, Educação, Esportes, Marketing, Clientes, Produtividade, Produtos, Saúde, Treinamentos e Vendas.

Dinâmica Restrições, Emoções, Narrativa, Progressão e Relacionamentos

$\begin{array}{ll}\text { Mecânica } & \begin{array}{l}\text { Desafios, Chance, Cooperação e competição, Avaliação (Feedback), Aquisição de } \\ \text { recursos, Recompensas, Transações, Turnos e Vitória }\end{array}\end{array}$

Conquistas, Avatar, Emblemas/medalhas, Boss, Coleções, Combate, Conteúdos

Componente desbloqueáveis, Presentes, Ranking, Níveis, Pontos, Missões, Gráfico Social, Times e Bens virtuais.

Fonte: Os autores

A análise do material coletado deu origem duas matrizes durante a etapa de codificação axial. Uma primeira matriz preliminar continha a variável segmento/área como sendo indicadora de categorias no eixo vertical, e os elementos de jogos no eixo horizontal. O preenchimento foi feito com o número de ocorrências de elemento de jogo em um determinado segmento/área. Mantiveram-se as categorias de elementos de jogos, e, as dez categorias (segmento/área) verificadas inicialmente, foram condensadas em quatro (sendo que a primeira - negócios - foi subdivida em subcategorias). Primeiramente, reuniram-se os conteúdos relacionados à negócios (Marketing, Clientes, Produtividade, Colaboratividade, Treinamentos 
Gamificação, elementos de jogos e estratégia: uma matriz de referência

e Vendas) em duas categorias: Negócio Externo e Interno. Considerou-se necessário descrever minimamente os segmentos/áreas (Quadro 6).

Quadro 6 - Descrição dos segmentos/Áreas identificados durante a Etapa de codificação axial

\begin{tabular}{|c|c|c|}
\hline \multicolumn{2}{|c|}{ Categorias } & Descrição \\
\hline \multirow{4}{*}{ Negócio } & $\begin{array}{l}(\text { Externo) } \\
\text { Marketing }\end{array}$ & $\begin{array}{l}\text { Marketing é um conjunto de processos voltados à criação, comunicação, } \\
\text { entrega e troca de ofertas que tenham valor para os clientes, consumidores, } \\
\text { parceiros e sociedade em geral (AMERICAN MARKETING } \\
\text { ASSOCIATION, c2014). } \\
\text { Obs: com base nesta definição foi possível anexar as categorias de clientes, produtos e vendas } \\
\text { à Negócio (Externo) - Marketing, uma vez que se englobam tais atividades. }\end{array}$ \\
\hline & $\begin{array}{l}\text { (Interno) } \\
\text { Treinamentos }\end{array}$ & $\begin{array}{l}\text { É um processo educacional, que possui uma aplicação sistêmica, através do } \\
\text { qual as pessoas aprendem conhecimentos, atitudes e habilidades em função } \\
\text { de objetivos definidos (CHIAVENATO, 2003) }\end{array}$ \\
\hline & $\begin{array}{c}\text { (Interno) } \\
\text { Colaboratividade }\end{array}$ & $\begin{array}{l}\text { A colaboratividade ocorre quando membros de um grupo, ao trabalharem } \\
\text { juntos, se apoiam visando atingir objetivos comuns para benefício geral do } \\
\text { grupo. (DAMIANI, 2008) }\end{array}$ \\
\hline & $\begin{array}{l}\text { (Interno) } \\
\text { Produtividade }\end{array}$ & $\begin{array}{l}\text { Entendida como uma unidade de produção sendo a relação entre quantidade } \\
\text { de produto e insumo (TUPY; YAMAGUCHI, 1998) }\end{array}$ \\
\hline \multicolumn{2}{|c|}{ Educação } & $\begin{array}{l}\text { [Para o contexto dessa pesquisa] Refere-se ao trabalho que se desenvolve } \\
\text { no escopo das unidades educacionais visando o processo ensino- } \\
\text { aprendizagem (DOURADO; OLIVEIRA, 2009) }\end{array}$ \\
\hline \multicolumn{2}{|c|}{ Saúde } & $\begin{array}{l}\text { Segmento que tem como finalidade a atenção à saúde humana visando um } \\
\text { estado de completo bem-estar físico, mental e social, e não apenas a } \\
\text { ausência de doenças (WORLD HEALTH ORGANIZATION, c2003) }\end{array}$ \\
\hline \multicolumn{2}{|c|}{ Esportes } & $\begin{array}{l}\text { Refere-se a tipos específicos de atividades que dependem das condições sob } \\
\text { as quais as atividades acontecem (BARBANTI, 2006) }\end{array}$ \\
\hline
\end{tabular}

Fonte: Os autores

Em uma segunda matriz (matriz final de referência) foram mantidas as mesmas categorias de segmento/área, tendo sido eliminados os elementos de jogos que não apresentaram ocorrências na primeira matriz, a saber: emoções, chance, transações, turnos, boss, coleções, combate e presentes. Para uma melhor visualização, utilizou-se o recurso de formatação condicional por intensidade de cor para destacar os elementos mais utilizados em um determinado segmento/área (Quadro 7). Vale ressaltar que a intenção da matriz é comparar 
os elementos dentro de cada categoria e não comparar os segmentos entre si, portanto mantevese o número de ocorrências e não relações de proporção entre os segmentos.

Quadro 7 -Elementos de jogos utilizados por Segmento/ Área

\begin{tabular}{|c|c|c|c|c|c|c|c|c|}
\hline & & $\begin{array}{l}\text { Negócio } \\
\text { (Externo) - } \\
\text { Marketing }\end{array}$ & $\begin{array}{c}\text { Negócio } \\
\text { (Interno) - } \\
\text { Treinamentos }\end{array}$ & $\begin{array}{c}\text { Negócio } \\
\text { (Interno) - } \\
\text { Colaboratividade }\end{array}$ & $\begin{array}{c}\text { Negócio } \\
\text { (Interno) - } \\
\text { Produtividade }\end{array}$ & Educação & Saúde & Esportes \\
\hline \multirow{3}{*}{ Dinâmica } & Narrativa & & 2 & & & 1 & & \\
\hline & Progressão & 11 & & 3 & & 10 & 5 & 3 \\
\hline & Relacionamentos & 9 & & 1 & & 5 & 1 & 3 \\
\hline \multirow{6}{*}{ Mecânica } & Desafios & 6 & 2 & 2 & 1 & 7 & 3 & 1 \\
\hline & $\begin{array}{l}\text { Cooperação e } \\
\text { competição }\end{array}$ & 3 & & 2 & 3 & 8 & 2 & 4 \\
\hline & Avaliação (Feedback) & 12 & & 2 & 2 & 7 & 4 & 3 \\
\hline & Aquisição de recursos & 2 & & & & & 1 & \\
\hline & Recompensas & 15 & & 4 & & 2 & 1 & 3 \\
\hline & Vitória & & 1 & & & 1 & 1 & 1 \\
\hline \multirow{10}{*}{ Componente } & Conquistas & 8 & 4 & 1 & 1 & 6 & 4 & 1 \\
\hline & Avatar & 1 & 1 & & 2 & 1 & & \\
\hline & Emblemas e medalhas & 12 & & 4 & 1 & 3 & 2 & 1 \\
\hline & Ranking & 3 & & 4 & 2 & 6 & 2 & 4 \\
\hline & Níveis & 6 & & & 1 & 4 & & \\
\hline & Pontos & 15 & 1 & 6 & 1 & 7 & 1 & 3 \\
\hline & Quests & 6 & & 2 & & 1 & & \\
\hline & Gráfico Social & 4 & & & 1 & 3 & 1 & 2 \\
\hline & Times & & & & 1 & 2 & & 1 \\
\hline & Bens virtuais & 2 & & 2 & & 2 & & \\
\hline
\end{tabular}

Fonte: Os autores

Importante ressaltar que, dos 61 casos coletados, as aplicações de gamificação relacionadas com a área de Negócios (Externos ou Internos) representam uma parcela de 59\% do total de casos coletados. A área de Educação é segunda que mais apresenta casos de gamificação, com $25 \%$ do total. Esportes e Saúde tiveram o mesmo número de casos, representando, cada um, $8 \%$ do total de casos.

Em seguida, a matriz foi separada em três blocos de maneira a permitir uma análise de dados por elementos de jogos. Inicialmente se apresenta a matriz de componentes (Quadro 8), que são os elementos mais concretos e visíveis do jogo. 
Gamificação, elementos de jogos e estratégia: uma matriz de referência

Quadro 8 - Componentes Mais Utilizados Por Segmento/Área

\begin{tabular}{|c|c|c|c|c|c|c|c|}
\hline & $\begin{array}{l}\text { Negócio } \\
\text { [Esterno] } \\
\text { Marketing }\end{array}$ & $\begin{array}{l}\text { Negócio } \\
\text { (Interno] } \\
\text { Treinamentos }\end{array}$ & $\begin{array}{c}\text { Negócio } \\
\text { (Interno) } \\
\text { Colaboratividade }\end{array}$ & $\begin{array}{l}\text { Negócio } \\
\text { (Interno) } \\
\text { Produtividade }\end{array}$ & Educação & Saúde & Esportes \\
\hline Conquistas & 8 & 4 & 1 & 1 & 6 & 4 & 1 \\
\hline Avatar & 1 & 1 & & 2 & 1 & & \\
\hline $\begin{array}{c}\text { Emblemas e } \\
\text { medalhas }\end{array}$ & 12 & & 4 & 1 & 3 & 2 & 1 \\
\hline \multicolumn{8}{|l|}{ Combate } \\
\hline Ranking & 3 & & 4 & 2 & 6 & 2 & 4 \\
\hline Níveis & 6 & & & 1 & 4 & & \\
\hline Pontos & 15 & 1 & 6 & 1 & 7 & 1 & 3 \\
\hline Quests & 6 & & 2 & & 1 & & \\
\hline $\begin{array}{l}\text { Gráfico } \\
\text { Social }\end{array}$ & 4 & & & 1 & 3 & 1 & 2 \\
\hline Times & & & & 1 & 2 & & 1 \\
\hline Bens virtuais & 2 & & 2 & & 2 & & \\
\hline
\end{tabular}

Fonte: Os autores

Quanto aos componentes mais utilizados, verifica-se a ênfase no uso da tríade de Pontos, Medalhas e Ranking. Embora a coleta não tenha identificado os três ocorrendo em conjunto, pelo menos um desses componentes surge em cada categoria. Pontos aparece três vezes como o componentes mais utilizado em Negócio (Externo) - Marketing; Negócio (Interno) Colaboratividade; e Educação. O Ranking é o componente mais utilizado em duas categorias: Negócio (Interno) - Produtividade; e Esportes. O componente Medalhas surge duas vezes em segundo lugar em Negócio (Externo) - Marketing; e Saúde, e uma vez (em terceiro lugar) em Negócio (Interno) - Colaboratividade.

Alguns componentes inesperados surgiram da coleta como, por exemplo, a Conquista que aparece como o componente mais utilizado em Negócio (Interno) - Treinamentos; e Saúde, e surge em terceiro em Negócio (Externo) - Marketing; e Educação. Outro elemento inesperado foi Avatar, que se manteve em segundo lugar em Negócio (Interno) - Produtividade; e Negócio (Interno) - Colaboratividade.

Ao se considerar que parte significativa dos casos relatavam aplicações na área de negócios, o processo de integração de elementos de jogo voltado a este segmento tem ênfase em campanhas de marketing, normalmente com o objetivo de estimular e motivar a participação e engajamento de usuários/clientes.

Ao se definirem os componentes é possível identificar diferentes mecânicas. Ou seja, os componentes são uma forma de atingir uma ou mais mecânicas. Como resultado da análise, o 
Quadro 9, lista as mecânicas mais utilizadas por segmento/área.

Quadro 9 - Mecânicas mais utilizadas por Segmento/Área

\begin{tabular}{|c|c|c|c|c|c|c|c|}
\hline & $\begin{array}{l}\text { Negócio } \\
\text { [Esterno] } \\
\text { Marketing }\end{array}$ & $\begin{array}{l}\text { Negócio } \\
\text { (Interno) } \\
\text { Treinamentos }\end{array}$ & \begin{tabular}{|c|}
$\begin{array}{c}\text { Negócio } \\
\text { (Interno) } \\
\text { Colaboratividade }\end{array}$ \\
\end{tabular} & $\begin{array}{c}\text { Negócio } \\
\text { (Interno) } \\
\text { Produtividade }\end{array}$ & Educạ̧ão & Saúde & Esportes \\
\hline Desafios & 6 & 2 & 2 & 1 & 7 & 3 & 1 \\
\hline $\begin{array}{c}\text { Cooperação e } \\
\text { competiçâao }\end{array}$ & 3 & & 2 & 3 & 8 & 2 & 4 \\
\hline $\begin{array}{c}\text { Avaliação } \\
\text { [Feedback] }\end{array}$ & 12 & & 2 & 2 & 7 & 4 & 3 \\
\hline $\begin{array}{l}\text { Aquisição de } \\
\text { recursos }\end{array}$ & 2 & & & & & 1 & \\
\hline Recompensas & 15 & & 4 & & 2 & 1 & 3 \\
\hline Vitória & & 1 & & & 1 & 1 & 1 \\
\hline
\end{tabular}

Fonte: Os autores

As mecânicas mais utilizadas variaram pouco de segmento para segmento - assim como ocorreu com os componentes - devido ao fato de tais elementos podem estar ligados a uma ou mais mecânicas. Portanto, ao se repetir o componente há uma probabilidade de se repetir também a mecânica. Negócio (Externo) - Marketing; e Negócio (Interno) - Colaboratividade apresentaram mais ocorrências com Recompensas; enquanto que Negócio (Interno) Treinamento relacionou-se com a mecânica Desafios. As mecânicas Cooperação e Competição destacaram-se no segmento Negócio (Interno) - Produtividade; Educação; e Esportes, sendo que Avaliação (Feedback) apareceu mais frequentemente no segmento de Saúde.

As Recompensas são os benefícios que os usuários recebem por alguma ação ou realização a partir de uma conquista no jogo. A utilização dessa mecânica nos segmentos de Negócio (Externo) - Marketing; e Negócio (Interno) - Colaboratividade pode se beneficiar do uso dos seguintes componentes: Emblemas e Medalhas, Bens Virtuais e Pontos. Os Desafios se caracterizam por serem os objetivos que o jogo define para o jogador. Assim, o uso de Desafios em Negócio (Interno) - Treinamento; e de Avaliação (Feedback) em Saúde podem estar relacionados ao componente Conquistas que os jogadores recebem ao cumprirem com um desafio no jogo. Cooperação e Competição trazem uma noção de vitória e derrota ao jogador e, ao serem usadas em Negócio (Interno) - Produtividade; em Educação, e em Esportes podem estar ligadas a noção que o componente Ranking ressalta aqueles jogadores mais bem sucedidos nos jogos.

Assim como componentes dão suporte a mecânicas, as mecânicas estão diretamente ligadas à dinâmicas, as quais representam o nível mais abstrato dos elementos de jogos (Quadro $10)$. 
Gamificação, elementos de jogos e estratégia: uma matriz de referência

Quadro 10 - Dinâmicas mais utilizadas por Segmento/Área

\begin{tabular}{|c|c|c|c|c|c|c|c|}
\cline { 2 - 8 } \multicolumn{1}{c|}{} & $\begin{array}{c}\text { Negócio } \\
\text { (Externo) } \\
\text { Marketing }\end{array}$ & $\begin{array}{c}\text { Negócio } \\
\text { (Interno) } \\
\text { Treinamentos }\end{array}$ & $\begin{array}{c}\text { Negócio } \\
\text { (Interno) } \\
\text { Colaboratividade }\end{array}$ & $\begin{array}{c}\text { Negócio } \\
\text { (Interno) } \\
\text { Produtividade }\end{array}$ & Educação & Saúde & Esportes \\
\hline Narrativa & & 2 & & & $\mathbf{1}$ & & \\
\hline Progressão & $\mathbf{1 1}$ & & $\mathbf{3}$ & & $\mathbf{5}$ & $\mathbf{3}$ & $\mathbf{3}$ \\
\hline Relacionamentos & $\mathbf{9}$ & & $\mathbf{1}$ & & $\mathbf{5}$ & $\mathbf{3}$ & $\mathbf{3}$ \\
\hline
\end{tabular}

Fonte: Os Autores

Nota-se que a dinâmica de Progressão é predominante em quatro segmentos/áreas (Negócio Externo - Marketing; Negócio Interno - Colaboratividade; Educação; e Saúde). Esta dinâmica transmite aos jogadores a sensação de avançar no jogo, pois a ideia de evolução parece ser mais efetiva em aplicações de gamification quanto comparadas a Narrativas e Relacionamentos. A dinâmica de Relacionamentos acarreta uma interação entre os usuários que podem ser companheiros ou adversários - o que para o segmento de Esportes pode ser eficaz para objetivos voltados à competição e cooperação.

As Narrativas, mais utilizadas no segmento de Negócio Interno - Treinamentos provavelmente são escolhidas para transmitir uma sequência lógica do que se quer ensinar aos funcionários. No segmento Negócio (Interno) - Produtividade não há qualquer dinâmica explicita relatada nos casos, embora as aplicações utilizem componentes de Ranking, Avatar, e mecânicas de Competição e Cooperação.

\section{Considerações finais}

A combinação entre componentes, mecânicas e dinâmicas pode ser distinta entre os diferentes segmentos mesmo que estes tenham elementos em comum. Na comparação entre o segmento Negócio (Externo) - Marketing, e a área de Educação, por exemplo, a combinação de elementos de jogos é - para o primeiro - Pontos > Recompensas > Progressão e, para a segunda, Pontos > Cooperação e competição > Progressão. Ou seja, mesmo que o componente e a dinâmica sejam utilizados em distintas áreas, a mecânica é diferente. No contexto da arquitetura de informação, e mais especificamente, dos aspectos lúdicos e de experiência sensorial característicos do engajamento, este é um dos aspectos que pode mudar a forma como o usuário final perceberá e se envolverá com o game, uma vez que são as mecânicas que "montam" o estilo do jogo. 
Considerando-se que os elementos de jogos podem ser combinados de diversas maneiras e cada segmento/área apresenta um potencial de combinações diferentes, é razoável supor que cada objetivo de negócio leva a relações distintas de elementos de jogos. As combinações podem estar alinhadas com as características e objetivos de cada segmento/área como, por exemplo, o uso de Narrativas em Educação e Negócio Interno - Treinamentos, devido ao estímulo, motivação e engajamento que estas podem promover no processo de ensino/aprendizagem.

Os desenvolvedores de gamificação devem, portanto, planejar com antecedência e escolher seus elementos de jogos com base nos objetivos de negócio (característica estratégica da gamificação), ou seja: qual a razão para gamificar os produtos e serviços? Como a gamificação irá beneficiar os usuários/clientes? Quais são os comportamentos desejados dos usuários/clientes? E, como levar os usuários a cumprirem com os objetivos estabelecidos? Quanto mais informações são coletadas sobre o negócio, maior é a chance de se projetar uma experiência eficaz e relevante de gamificação. Definidas essas questões, a matriz derivada da pesquisa pode auxiliar na escolha dos elementos mais adequados ao contexto e objetivo de negócio.

Ainda que não explorado diretamente neste estudo, um segundo momento estratégico está relacionado com a coleta de informação advinda das atividades dos usuários de gamificação. Métricas relativas aos resultados da atividade efetiva de jogo podem auxiliar a organização a verificar se os objetivos pretendidos foram atingidos e, consequentemente, o grau de sucesso da aplicação. O número usuários ativos diários em relação a usuários ativos mensais, o tempo gasto na aplicação, a ação dos usuários na aplicação, os usuários retidos, e número de inscritos, por exemplo, são indicadores da efetividade da gamificação no contexto estratégico da organização. As informações daí derivadas permitiriam o entendimento do perfil do público alvo, a identificação de padrões e a compreensão de quais tipos de dinâmicas, mecânicas e componentes de jogo impulsionariam o comportamento desejado. Ao se explorar o processo de tomada de decisão, os implementadores podem - com base nos dados gerados nas atividades dos jogadores - planejar de forma mais efetiva e incentivar tanto o uso dos produtos e serviços, como o aumento da visibilidade da marca e o reforço dos comportamentos desejados; indispensáveis para o sucesso da organização.

Vale ressaltar que desenvolvedores respeitados, tais como a Bunchball Incorporation e Badgeville, têm plataformas de gamificação pré-definidas, o que pode influenciar o uso de 
determinados tipos de elementos de jogos (sendo a tríade de componentes Pontos, Ranking e Medalhas a mais comum). Portanto, é razoável supor que a escolha dos elementos seja também influenciada pelas ferramentas e plataformas disponíveis para o desenvolvimento destas aplicações, ocultando a possibilidade da utilização de outros elementos. Esta seria uma abordagem a ser explorada em futuros estudos na temática.

Ainda que o estudo não tenha enfocado os tipos de jogadores, a estrutura da matriz foi desenvolvida para permitir flexibilidade e a adição de novos segmentos e elementos de jogos, podendo ainda ser explorada utilizando-se os elementos de jogos mais utilizados por segmento/área em relação aos tipos de jogadores e suas motivações específicas.

\section{Referências}

AMERICAN MARKETING ASSOCIATION. Definition of marketing. c2014. Disponível em: 〈https://www.ama.org/AboutAMA/Pages/Definition-of-Marketing.aspx >. Acesso em: 26 jan. 2015 .

BARBANTI, V. O que é esporte? Revista Brasileira de Atividade Física \& Saúde, v. 11, n. 1, 2006. Disponível em:

<http://periodicos.ufpel.edu.br/ojs2/index.php/RBAFS/article/view/833/840>. Acesso em: 2 set. 2015.

BUNCHBALL INC. Gamification 101: an introduction to the use of game dynamics to influence behavior. 2010. Disponível em:

$<$ http://www.bunchball.com/sites/default/files/downloads/gamification101.pdf $>$. Acesso em: 02 set. 2015.

The white paper: enterprise gamification, the gen Y factor. 2012. Disponível em: $<$ http://www.gamification.co/wp-content/uploads/gettingstarted/White\%20Paper_Enterprise\%20Gamification_The_Gen_Y_Factor_2012.pdf >. Acesso em: 26 jan. 2015.

CHARMAZ, K. Constructing grounded theory: a practical guide through qualitative analysis. London: Sage, 2006

CHIAVENATO, I. Introdução à teoria geral da administração. Rio de Janeiro: Elsevier, 2003.

CORBIN, J.; STRAUSS, A. Grounded Theory research: procedures, canons, and evaluative criteria. Ebsco Publishing, v. 13, n. 1, p.3-20, dez. 1990. Disponível em: $<$ https://wiki.umn.edu/pub/WrittenArguments/Resources/Corbin_Strauss__Grounded_theory_Research.pdf $>$. Acesso em: 26 jan. 2015. 
CUCCUREDDU, G. Gartner's hype cycle 2011: social analytics and activity streams reach "the peak". Business Insider. Aug. 11, 2011. Disponível em:

$<$ http://www.businessinsider.com/gartners-hype-cycle-2011-social-analytics-and-activitystreams-reach-the-peak-2011-8>. Acesso em: 22 jan. 2015.

DAMIANI, M. F. Entendendo o trabalho colaborativo em educação e revelando seus benefícios. Educar, Curitiba, n. 31, p.213-230, maio 2008.

DETERDING, S. Gamification: designing for motivation. Interactions, v.19, n. 4, p. 14-17, jul./ago., 2012. Disponível em: 〈http://dx.doi.org/10.1145/2212877.2212883>. Acesso em: 26 jan. 2015.

. Situated motivational affordances of game elements: a conceptual model. In: WORKSHOP ON GAMIFICATION: USING GAME DESIGN ELEMENTS IN NONGAMING CONTEXTS, 2011, Vancouver, Canadá. Proceedings... Vancouver, 2011. p. 1-4 Disponível em: <http://gamification-research.org/wp-content/uploads/2011/04/09-

Deterding.pdf>. Acesso em: 26 jan. 2015.

et al. Designing gamification: creating gameful and playful experiences. In: Conference on Human Factors in Computing Systems, 2013, Paris, França. Proceedings... Paris, 2013. p. 1-4. Disponível em: 〈http://gamification-research.org/wpcontent/uploads/2013/03/20.Gamification_Extended_Abstract.pdf $>$. Acesso em: 26 jan. 2015.

Gamification: using game design elements in non-gaming contexts. In: CONFERENCE ON HUMAN FACTORS IN COMPUTING SYSTEMS, 2011, Vancouver, Canada. Proceedings... Vancouver, 2011. p. 1-4. Disponível em: <http://gamificationresearch.org/wp-content/uploads/2011/04/01-Deterding-Sicart-Nacke-OHara-Dixon.pdf >. Acesso em: 26 jan. 2015.

DOURADO, L; OLIVEIRA, F. A qualidade da educação: perspectivas e desafios. Cedes, Campinas, v. 29, n. 78, p.201-215, maio 2009. Disponível em: <http://www.scielo.br/pdf/ccedes/v29n78/v29n78a04>. Acesso em: 26 jan. 2015.

ELLIS, D.A. Behavioral approach to information retrieval system design, Journal of Documentation, v. 45, n. 3, p. 171-212, 1989. Disponível em: <http://dx.doi.org/10.1108/eb026843>. Acesso em: 26 jan. 2015.

ERIKSSON, B; MUSIALIK, M; WAGNER, J. Gamification engaging the future. 2012. 18f. Bachelor Thesis - Department of Computer Science and Engineering, University Of Gothenburg, Gothenburg, 2012. Disponível em: <https://gupea.ub.gu.se/bitstream/2077/30037/1/gupea_2077_30037_1.pdf>. Acesso em: 26 jan. 2015.

FAHEY, F. GDC: Nintendo announces DS online, first revolution details. Eurogamer.net. 2005. Disponível em: <http://www.eurogamer.net/articles/news_100305_GDCNintendoIwata>. Acesso em: 16 maio 2014. 
GARRIS, R.; AHLERS, R.; DRISKELL, J. E. Games, motivation, and learning: a research and practice model. Simulation \& Gaming, v. 33, n. 4, p. 441-467, Dec. 2002. Disponível em: 〈http://dx.doi.org/10.1177/1046878102238607>. Acesso em: 20 ago. 2015.

GARTNER GROUP. 2011 Hype cycles. 2011. Disponível em:

<http://www.gartner.com/technology/research/methodologies/hype-cycle.jsp >. Acesso em: 22 jan. 2015.

Gartner says by 2014,80 percent of current gamified applications will fail to meet business objectives primarily due to poor design. 2012. Disponível em: <http://www.gartner.com/newsroom/id/2251015 >. Acesso em: 22 jan. 2015.

INSTITUTO BRASILEIRO DE GEOGRAFIA E ESTATÍSTICA. Introdução à classificação nacional de atividades econômicas: CNAE versão 2.0. 2007. Disponível em: $<$ http://concla.ibge.gov.br/images/concla/documentacao/CNAE20_Introducao.pdf>. Acesso em: 26 jan. 2015.

KUUTTI, J. Designing gamification. 68 f. Dissertação (Master in Business Administration) - University of Oulo, Oulo, 2013. Disponível em: <http://herkules.oulu.fi/thesis/nbnfioulu201306061526.pdf>. Acesso em: 26 jan. 2015.

MARONEY, K. My entire waking life. 2001. Disponível em: $\langle$ http://www.thegamesjournal.com/articles/MyEntireWakingLife.shtml >. Acesso em: 26 jan. 2015.

MCCORMICK, T. Gamification: a short history. June, 2013. Disponível em:

<http://www.foreignpolicy.com/articles/2013/06/24/anthropology_of_an_idea_gamification>. Acesso em: 26 jan. 2015.

NICHOLSON, S. A user-centered theoretical framework for meaningful gamification. In: GAMES LEARNING SOCIETY 8.0, 2012, Madison, Wisconsin. Proceedings... Madison, 2012. p. 1-7. Disponível em: 〈http://scottnicholson.com/pubs/meaningfulframework.pdf〉. Acesso em: 26 jan. 2015.

O'BRIEN, H.; TOMS, E. What is user engagement? a conceptual framework for defining user engagement with technology. Journal of the American Society for Information Science and Technology, v. 59, n. 6, p. 938-955, 2008. Disponível em:

〈http://faculty.arts.ubc.ca/hobrien/files/OBrien_Toms_2008_What_is_user_engagement.pdf $>$ . Acesso em: 20 ago. 2015.

PRIEBATSCH, S. The game layer on top of the world. TED, Boston, 2010. Disponível em: $<$ http://www.ted.com/talks/seth_priebatsch the_game_layer_on_top_of_the_world $>$. Acesso em: 26 jan. 2015.

QUESENBERY, W. Dimensions of usability: opening the conversation, driving the process. In: USABILITY PROFESSIONALS' ASSOCIATION ANNUAL CONFERENCE. Proceedings..., 2003, Scottsdale, Arizona, p.1-8. Disponível em: 〈http://www.wqusability.com/articles/5es-upa2003.pdf>. Acesso em: 26 ago. 2015. 
SCHÖNEN, R. Gamification in change management processes: an empirical research by means of qualitative methods to analyze relevance, implications and selected use cases. 2014. 79 f. Bachelor Thesis - Applied Sciences, University of Applied Sciences, Munich, 2014.

Disponível em: <http://enterprisegamification.com/attachments/article/196/BA\%20Thesis\%20\%20Gamification\%20v1.0.3_Final.pdf>. Acesso em: 26 jan. 2015.

TOMS, E.G. Information interaction: providing a framework for information architecture. Journal of the American Society for Information Science and Technology, v. 53, n. 10, p. 855-862, 2002. Disponível em: 〈https://www.unc.edu/ acrystal/110-117/toms.pdf>. Acesso em: 20 ago. 2015.

TUPY, O; YAMAGUCHI, T. Eficiência e produtividade: conceitos e medição. Agricultura em São Paulo, São Paulo, v. 51, n. 39, p.39-51, 1998. Disponível em: <ftp://ftp.sp.gov.br/ftpiea/rea/tomo2_98/artigo3.pdf>. Acesso em: 26 jan. 2015.

WERBACH, K.; HUNTER, D. For the win: how game thinking can revolutionize your business. Philadelphia: Wharton Digital Press, 2012.

WORLD HEALTH ORGANIZATION. WHO definition of Health. c2003. Disponível em: <http://www.who.int/about/definition/en/print.html>. Acesso em: 02 set. 2015.

ZICHERMANN, G.; CUNNINGHAM, C. Gamification by design. Sebastopol: O’Reilly, 2011. 